TRANSACTIONS OF THE

AMERICAN MATHEMATICAL SOCIETY

Volume 349, Number 1, January 1997, Pages 235-255

S 0002-9947(97)01644-9

\title{
BOUNDEDNESS OF THE FRACTIONAL INTEGRAL ON WEIGHTED LEBESGUE AND LIPSCHITZ SPACES
}

\author{
ELEONOR HARBOURE, OSCAR SALINAS, AND BEATRIZ VIVIANI
}

\begin{abstract}
Necessary and sufficient conditions are given for the fractional integral operator $I_{\alpha}$ to be bounded from weighted strong and weak $L^{p}$ spaces within the range $p \geq n / \alpha$ into suitable weighted $B M O$ and Lipschitz spaces. We also characterize the weights for which $I_{\alpha}$ can be extended to a bounded operator from weighted $B M O$ into a weighted Lipschitz space of order $\alpha$. Finally, under an additional assumption on the weight, we obtain necessary and sufficient conditions for the boundedness of $I_{\alpha}$ between weighted Lipschitz spaces.
\end{abstract}

\section{INTRODUCTION}

A nonnegative function $\omega$ defined on $\mathbb{R}^{n}$ is called a weight if it is locally integrable. We denote by $|E|$ the Lebesgue measure of $E$, and $\omega(E)=\int_{E} \omega(x) d x$. Given a ball $B, \theta B$ will mean the ball with the same center as $B$ and with radius $\theta$ times as long. Throughout this paper, the letter $C$ will denote a constant not necessarily the same at each occurrence.

A weight $\omega$ is said to belong to the Muckenhoupt class $A_{p}, 1<p<\infty$, if there exists a constant $C$ such that

$$
\frac{\omega(B)}{|B|}\left(\frac{\omega^{-\frac{1}{p-1}}(B)}{|B|}\right)^{p-1} \leq C
$$

for every ball $B \subset \mathbb{R}^{n}$. The class $A_{1}$ is defined replacing the above inequality by

$$
\left\|\omega^{-1} \mathcal{X}_{B}\right\|_{\infty} \frac{\omega(B)}{|B|} \leq C,
$$

where $\mathcal{X}_{B}$ is the characteristic function of the ball $B$. We shall say that a weight $w$ satisfies a doubling condition if there exists a constant $C$ such that

$$
\omega(2 B) \leq C \omega(B)
$$

Received by the editors June 26, 1995.

1991 Mathematics Subject Classification. Primary 42B25.

Key words and phrases. Fractional integral, weighted Lebesgue and Lipschitz spaces, weighted BMO.

The authors were supported by the Consejo Nacional de Investigaciones Científicas y Técnicas de la República Argentina and by the Universidad Nacional del Litoral, CAI+D Program.

(C)1997 American Mathematical Society 
for every ball $B \subset \mathbb{R}^{n}$. Given $p>1$, we shall say that $\omega \in R H(p)$, if $\omega$ satisfies a reverse Hölder condition with exponent $p$, that is,

for every ball $B \subset \mathbb{R}^{n}$.

$$
\left(\frac{\omega^{p}(B)}{|B|}\right)^{1 / p} \leq C \frac{\omega(B)}{|B|}
$$

By $L^{p}$ and $\widetilde{L}^{p}$ we mean the usual strong and weak Lebesgue spaces on $\mathbb{R}^{n}$, and we denote by $\|\cdot\|_{p}$, respectively $[\cdot]_{p}$, the corresponding norms, that is,

$$
\|f\|_{p}^{p}=\int_{\mathbb{R}^{n}}|f(x)|^{p} d x \quad \text { and } \quad[f]_{p}^{p}=\sup _{t>0} t^{p}|\{x / f(x)>t\}| .
$$

We shall denote by $L_{w}^{p}$ the class of functions $f$ such that $\|f\|_{p, \omega} \equiv\|f / \omega\|_{p}$ is finite. Similarly, we shall say that $f$ belongs to $\widetilde{L}_{\omega}^{p}$ if $[f]_{p, \omega}=[f / \omega]_{p}$ is finite. Finally, the Lipschitz space $\Lambda(\delta), 0<\delta<1$, is the class of functions $f$ satisfying $|f(x)-f(y)| \leq C|x-y|^{\delta}$.

We consider the fractional integral operator $I_{\alpha}, 0<\alpha<n$, defined by

$$
I_{\alpha} f(x)=\int_{\mathbb{R}^{n}} f(y)|x-y|^{\alpha-n} d y
$$

whenever this integral is finite. Clearly, if $f$ belongs to $L_{c}^{\infty}=\{g: g$ is a bounded function with compact support $\}$, then the integral in (0.1) is finite for every $x$. Moreover, for $f$ in $L_{c, \omega}^{\infty}$, that is $f / \omega \in L_{c}^{\infty}$, it is easy to check that the integral in (0.1) is finite for almost every $x \in \mathbb{R}^{n}$. In fact, letting $B_{0}=B\left(0, R_{0}\right)$ be such that supp $f \subset B_{0}$ and $R>0$, we have

$$
\begin{aligned}
\int_{B(0, R)}\left|I_{\alpha} f(x)\right| d x & \leq\|f / \omega\|_{\infty} \int_{B(0, R)}\left(\int_{B_{0}} \omega(y)|x-y|^{\alpha-n} d y\right) d x \\
& \leq\|f / \omega\|_{\infty} \int_{B_{0}} \omega(y)\left(\int_{B\left(x, R_{0}+R\right)} \frac{d x}{|x-y|^{n-\alpha}}\right) d y \\
& \leq C\left(\alpha, R_{0}, R\right) \omega\left(B_{0}\right)\|f / \omega\|_{\infty} .
\end{aligned}
$$

Thus, $I_{\alpha} f(x)$ is finite for almost every $x \in B(0, R)$, and, consequently, for almost every $x \in \mathbb{R}^{n}$.

For $1<p<n / \alpha$ and $1 / q=1 / p-\alpha / n$, Muckenhoupt and Wheeden in [M-W1] characterize the non negative functions $v$ for which the inequality

$$
\left(\int\left|I_{\alpha} f\right|^{q} v^{-q}\right)^{1 / q} \leq C\left(\int|f|^{p} v^{-p}\right)^{1 / p}
$$

holds, as those belonging to the class $A(p, q)$, i.e.

$$
\left(\frac{v^{-q}(B)}{|B|}\right)^{1 / q}\left(\frac{v^{p \prime}(B)}{|B|}\right)^{1 / p \prime} \leq C
$$

holds for any ball $B \subset \mathbb{R}^{n}$ and $p^{\prime}=p /(p-1)$. It is easy to see that $v \in A(p, q)$ if and only if $v^{p^{\prime}} \in A_{1+p^{\prime} / q}$. For the limiting case $p=n / \alpha$ and $q=\infty$, they also 
characterize the weights satisfying the inequality

$$
\left\|v^{-1} \mathcal{X}_{B}\right\|_{\infty} \frac{1}{|B|} \int_{B}\left|I_{\alpha} f(x)-m_{Q}\left(I_{\alpha} f\right)\right| d x \leq C\|f\|_{n / \alpha, v},
$$

as the weights belonging to $A(n / \alpha, \infty)$, that is, $v^{(n / \alpha)^{\prime}} \in A_{1}$. Here $m_{Q}(g)$ denotes the average $\frac{1}{|Q|} \int_{Q} g(x) d x$. This inequality may be viewed as the boundedness of $I_{\alpha}$ from $L_{n / \alpha, v}$ into a weighted version of $B M O$, the space of functions with bounded mean oscillation.

In this work we give necessary and sufficient conditions on the weights for the boundedness of the fractional integral operator $I_{\alpha}$ from weighted $L_{\omega}^{p}$ and $\widetilde{L}_{\omega}^{p}, n / \alpha \leq$ $p<n /(\alpha-1)^{+}$, into suitable weighted $B M O$ and Lipschitz spaces. Actually, for the case $p=n / \alpha$ a slightly more general result is obtained here than that stated in (0.3) (see Remark 2.15). We also and the boundedness characterize the weights for which $I_{\alpha}$ can be extended to a bounded operator from weighted $B M O$ into a weighted Lipschitz space of order $\alpha$. Finally, under an additional assumption on the weight, we obtain necessary and sufficient conditions for the boundedness of $I_{\alpha}$ between weighted Lipschitz spaces. For the unweighted case, the results contained in this paper have been established in different settings by several authors, see for instance $[\mathrm{Z}],[\mathrm{S}-\mathrm{Z}],[\mathrm{G}-\mathrm{V}]$ and $[\mathrm{H}-\mathrm{S}-\mathrm{V}]$.

The paper is organized as follows. In $\S 1$ we define the family of spaces used throughout the work and derive some basic properties. The main results of the paper, concerning the boundedness of fractional integrals, are stated in $\S 2$. In $\S 3$, we study the basic properties of the class of weights related to the inequalities given in $\S 2$. Finally, in $\S 4$, we prove the weighted inequalities stated in $\S 2$.

\section{The SPACES $\mathcal{L}_{\omega}(\beta)$ AND $\overline{\mathcal{L}}_{\omega}(\beta)$}

We start by giving two possible weighted versions of the spaces $\mathcal{L}_{p, \lambda}$, for $p=1$, considered by Peetre in $[\mathrm{P}]$.

(1.1) Definition. Let $\omega$ be a weight and $-1<\beta<1 / n$. We say that a locally integrable function $f$ belongs to $\mathcal{L}_{\omega}(\beta)$ if there exists a constant $C$ such that

$$
\frac{1}{\omega(B)|B|^{\beta}} \int_{B}\left|f(x)-m_{B} f\right| d x \leq C
$$

for every ball $B \subset \mathbb{R}^{n}$. The least constant $C$ satisfying this inequality will be denoted by $\|f\|_{\mathcal{L}_{\omega}(\beta)}$.

(1.2) Definition. Let $-1<\beta<1 / n$, and let $\omega$ be a weight. We denote by $\overline{\mathcal{L}}_{\omega}(\beta)$ the space of the locally integrable functions $f$ such that the inequality

$$
\frac{1}{\left(\omega^{\frac{1}{1+\beta}}(B)\right)^{1+\beta}} \int_{B}\left|f(x)-m_{B} f\right| d x \leq C
$$

holds for a fixed constant $C$ and for every ball $B \subset \mathbb{R}^{n}$. The least constant $C$ will be called $\|f\|_{\overline{\mathcal{L}}_{\omega}(\beta)}$.

Let us observe that for $\beta=0$, both of the spaces $\mathcal{L}_{\omega}(\beta)$ and $\overline{\mathcal{L}}_{\omega}(\beta)$ coincide with one of the versions of weighted bounded mean oscilation space, introduced by Muckenhoupt and Wheeden in [M-W2]. Moreover, for the case $\omega \equiv 1$, both Definitions 1.1 and 1.2 give the known Lipschitz integral spaces for $\beta$ in the range 
$0<\beta<1 / n$, and the Morrey spaces, for $-1<\beta<0$. Also, we notice that the spaces $\overline{\mathcal{L}}(\beta), 0 \leq \beta<1 / n$, are the duals of the weighted Hardy spaces $H_{\omega^{p}}^{p}$ with $p=\frac{1}{1+\beta}$, introduced by García Cuerva in $[\mathrm{GC}]$.

The following propositions establish the relationships between the spaces $\mathcal{L}_{\omega}(\beta)$ and $\overline{\mathcal{L}}_{\omega}(\beta)$ and some useful properties of them.

(1.3) Proposition. Let $0<\beta<1 / n$, and let $\omega$ be a weight. Then

(1.4) The space $\overline{\mathcal{L}}_{\omega}(\beta)$ is contained in $\mathcal{L}_{\omega}(\beta)$. If $\omega^{1 /(1+\beta)} \in R H(1+\beta)$, then $\mathcal{L}_{\omega}(\beta) \equiv \overline{\mathcal{L}}_{\omega}(\beta)$.

(1.5) If $\omega$ satisfies a doubling condition, the space $\mathcal{L}_{\omega}(\beta)$ coincides with the pointwise version $\Lambda_{\omega}(n \beta)$ consisting of all the functions $f$ such that there exists a constant $C$ satisfying

$$
|f(x)-f(y)| \leq C\left(\int_{B(x, 2|x-y|)} \frac{\omega(z)}{|z-x|^{n-\beta n}} d z+\int_{B(y, 2|x-y|)} \frac{\omega(z)}{|z-y|^{n-\beta n}} d z\right)
$$

for almost every $x$ and $y$ in $\mathbb{R}^{n}$. In particular, for the case $\omega \equiv 1$, we obtain that $f \in \mathcal{L}_{\omega}(\beta)$ if and only if $f \in \Lambda(n \beta)$.

Proof. That $\overline{\mathcal{L}}_{\omega}(\beta) \subset \mathcal{L}_{\omega}(\beta)$ follows directly from the Hölder inequality, and the other inclusion is also inmediate from our assumption that $\omega^{1 /(1+\beta)} \in R H(1+\beta)$.

In order to prove (1.5), we first check (1.6) for $f$ in $\mathcal{L}_{\omega}(\beta)$. Given $x$ and $y$ in $\mathbb{R}^{n}, x \neq y$, take $B=B(x,|x-y|)$ and $B^{\prime}=B(y,|x-y|)$. Then

$$
|f(x)-f(y)| \leq\left|f(x)-m_{B} f\right|+\left|f(y)-m_{B^{\prime}} f\right|+\left|m_{B^{\prime}} f-m_{B} f\right| .
$$

We estimate only the first term of the right side, since the others follow similar lines. Letting $B_{i}=B\left(x, 2^{-i}|x-y|\right)$ for $i \geq 1$ and $B_{0}=B$, and using the doubling condition, we get

$$
\begin{aligned}
\mid f(x) & -m_{B} f \mid \leq \lim _{m \rightarrow \infty}\left(\left|f(x)-m_{B_{m}} f\right|+\sum_{i=0}^{m-1}\left|m_{B_{i+1}} f-m_{B_{i}} f\right|\right) \\
& \leq C \sum_{i=0}^{\infty}\left|B_{i}\right|^{-1} \int_{B_{i}}\left|f(z)-m_{B_{i}} f\right| d z \leq C|| f \|_{\mathcal{L}_{\omega}(\beta)} \sum_{i=0}^{\infty}\left|B_{i}\right|^{\beta-1} \omega\left(B_{i}\right) \\
& \leq C|| f||_{\mathcal{L}_{\omega}(\beta)} \sum_{i=0}^{\infty} \int_{B_{i}-B_{i+1}} \frac{\omega(z)}{|z-x|^{n-\beta n}} d z \\
& \leq C|| f \|_{\mathcal{L}_{\omega}(\beta)} \int_{B(x, 2|x-y|)} \frac{\omega(z)}{|z-x|^{n-\beta n}} d z,
\end{aligned}
$$

for almost every $x$ in $\mathbb{R}^{n}$.

Conversely, integrating (1.6) on a ball $B$ with respect to both variables, $x$ and $y$, and interchanging the order of integration, we obtain that $f$ belongs to $\mathcal{L}_{\omega}(\beta)$. This completes the proof of (1.5). $\diamond$

(1.8) Proposition. Let $\omega$ be a weight and $-1<\beta<0$. Then

(1.9) The space $\mathcal{L}_{\omega}(\beta)$ is contained in $\overline{\mathcal{L}}_{\omega}(\beta)$. If, in addition, $\omega \in R H(1 /(1+\beta))$, then $\mathcal{L}_{\omega}(\beta) \equiv \overline{\mathcal{L}}_{\omega}(\beta)$. 
The Lebesgue space $L_{\omega}^{-1 / \beta}$ is contained in $\overline{\mathcal{L}}_{\omega}(\beta)$.

Proof. First, let us prove (1.9). Since $0<1+\beta<1$, the inclusion of $\mathcal{L}_{\omega}(\beta)$ in $\overline{\mathcal{L}}_{\omega}(\beta)$ follows, as in (1.4), by using the Hölder inequality with exponent $1 /(1+\beta)$. The other inclusion is a consequence of the fact that $\omega \in R H(1 /(1+\beta))$. Now, let us show (1.10). Let $f \in L_{\omega}^{q}$, with $q=-1 / \beta$. The Hölder inequality implies that

$$
\begin{aligned}
\int_{B}\left|f(x)-m_{B} f\right| d x & \leq 2 \int_{B} \frac{|f(x)|}{\omega(x)} \omega(x) d x \\
& \leq 2\left(\int\left|\frac{f(x)}{\omega(x)}\right|^{q} d x\right)^{1 / q}\left(\int_{B} \omega(x)^{q^{\prime}} d x\right)^{1 / q^{\prime}} .
\end{aligned}
$$

Since $q^{\prime}=(-1 / \beta)^{\prime}=1 /(1+\beta)$, we get that

$$
\int_{B}\left|f(x)-m_{B} f\right| d x \leq 2\|f\|_{-1 / \beta, \omega}\left(\omega^{\frac{1}{1+\beta}}(B)\right)^{1+\beta},
$$

as we wanted to prove. $\diamond$

\section{Results on the Boundedness of $I_{\alpha}$}

We now introduce two classes of weights which appear in connection with the spaces $\mathcal{L}_{\omega}(\beta)$ and $\overline{\mathcal{L}}_{\omega}(\beta)$, and the boundedness of the operator $I_{\alpha}$.

(2.1) Definition. Let $0<\alpha<n$ and $1<p<\infty$. We say that $\omega \in H(\alpha, p)$ if there exists a constant $C$ such that

$$
|B|^{1 / p-\alpha / n+1 / n}\left(\int_{\mathbb{R}^{n}-B} \frac{\omega^{p^{\prime}}(y)}{\left|x_{B}-y\right|^{(n-\alpha+1) p^{\prime}}} d y\right)^{1 / p^{\prime}} \leq C \frac{\omega(B)}{|B|}
$$

for every ball $B \subset R$, where $x_{B}$ is the center of the ball $B$ and $p^{\prime}=p /(p-1)$. In the case $p=\infty,(2.2)$ should be understood to mean

$$
|B|^{-\alpha / n+1 / n} \int_{\mathbb{R}^{n}-B} \frac{\omega(y)}{\left|x_{B}-y\right|^{n-\alpha+1}} d y \leq C \frac{\omega(B)}{|B|} .
$$

We denote this class by $H(\alpha, \infty)$.

(2.3) Definition. Let $0<\alpha<n, 1<p<\infty$ and $\beta=\alpha / n-1 / p$. We define $\bar{H}(\alpha, p)$ as the class of weights $\omega$ such that there exists a constant $C$ satisfying

$$
|B|^{1 / p-\alpha / n+1 / n}\left(\int_{\mathbb{R}^{n}-B} \frac{\omega^{p^{\prime}}(y)}{\left|x_{B}-y\right|^{(n-\alpha+1) p \prime}} d y\right)^{1 / p^{\prime}} \leq C\left(\frac{\omega^{\frac{1}{1+\beta}}(B)}{|B|}\right)^{1+\beta}
$$

for every ball $B=B\left(x_{B}, R\right)$ in $\mathbb{R}^{n}$ and $p^{\prime}=p /(p-1)$. The class $\bar{H}(\alpha, \infty)$ is defined by taking $1 / p=0, p^{\prime}=1$ and $\beta=\alpha / n$ in (2.4).

In the case $n / \alpha<p \leq \infty$, by using Hölder inequality, it is easy to see that $\bar{H}(\alpha, p) \subseteq H(\alpha, p)$. Moreover, if $\omega^{1 /(1+\beta)} \in R H(1+\beta)$ with $\beta=\alpha / n-1 / p$, both classes are the same. In a similar way, when $1<p<n / \alpha$, we clearly have that $H(\alpha, p) \subseteq \bar{H}(\alpha, p)$, and both classes coincide whenever $\omega \in R H(1 /(1+\beta))$.

We also note that for $p=\infty$ and $n=1$, the class $H(\alpha, \infty)$ agrees with one of the classes $B_{r}$ considered by Muckenhoupt in [M] and earlier in [H-M-W]. It is known 
(see $[\mathrm{F}-\mathrm{M}]$ ) that there exist weights in $B_{r}$ which are not in $A_{\infty}$ and, consequently, weights in $B_{r}$ do not necessarily satisfy any reverse-Hölder condition.

Even though we will restrict our attention in this paper to the boundedness of $I_{\alpha}$ involving the spaces $\mathcal{L}_{\omega}(\beta)$ and the corresponding classes $H(\alpha, p)$, similar results to those stated in Theorem 2.5 below can be obtained for the spaces $\overline{\mathcal{L}}(\beta)$ and the classes $\bar{H}(\alpha, p)$.

Now we are in the position to state our main results

(2.5) Theorem. Let $0<\alpha<n, 1<p<n /(\alpha-1)^{+}$and $\beta=\alpha / n-1 / p$. The following statements are equivalent:

(2.6) The operator $I_{\alpha}$ can be extended to a bounded linear operator $\widetilde{I}_{\alpha}$ from $L_{\omega}^{p}$ into $\mathcal{L}_{\omega}(\beta)$ by means of

$$
\tilde{I}_{\alpha} f(x)=\int_{\mathbb{R}^{n}}\left[\frac{1}{|x-y|^{n-\alpha}}-\frac{1-\chi_{B(0,1)}(y)}{|y|^{n-\alpha}}\right] f(y) d y .
$$

(2.7) The operator $I_{\alpha}$ can be extended to a bounded linear operator $\widetilde{I}_{\alpha}$ from $\widetilde{L}_{\omega}^{p}$ into $\mathcal{L}_{\omega}(\beta)$, where $\widetilde{I}_{\alpha}$ is defined as in $(2.6)$.

(2.8) The weight $\omega$ belongs to $H(\alpha, p)$.

(2.9) Theorem. Let $\omega$ be a weight and $0<\alpha<1$. The following conditions are equivalent:

(2.10) The operator $I_{\alpha}$ can be extended to a bounded linear operator $\widetilde{I}_{\alpha}$ from $\mathcal{L}_{\omega}(0)$ into $\mathcal{L}_{\omega}(\alpha / n)$ by means of

$$
\tilde{I}_{\alpha} f(x)=\int_{\mathbb{R}^{n}}\left(\frac{1}{\left|x_{0}-y\right|^{n-\alpha}}-\frac{1}{|x-y|^{n-\alpha}}\right) f(y) d y
$$

for an appropiate choice of $x_{0} \in \mathbb{R}^{n}$.

(2.11) The weight $\omega$ belongs to $H(\alpha, \infty)$.

(2.12) Corollary. Let $\alpha, \delta \in \mathbb{R}^{+}$such that $0<\alpha+\delta<1$. The following conditions are equivalent:

(2.13) The weight $\omega$ belongs to $H(\delta, \infty)$ and the operator $I_{\alpha}$ can be extended to a bounded linear operator $\widetilde{I}_{\alpha}$ from $\mathcal{L}_{\omega}(\delta / n)$ into $\mathcal{L}_{\omega}((\alpha+\delta) / n)$.

(2.14) The weight $\omega$ belongs to $H(\alpha+\delta, \infty)$.

(2.15) Remark. We note that for $\omega \equiv 1$, Theorem 2.5 gives, in particular, the classical results:

$$
I_{\alpha}: \widetilde{L}^{p} \rightarrow \Lambda(\beta), \quad \frac{n}{\alpha}<p<\frac{n}{(\alpha-1)^{+}},
$$

and

$$
I_{\alpha}: \widetilde{L}^{n / \alpha} \rightarrow B M O
$$

For general $\omega$ and $p=n / \alpha$ (i.e. $\beta=0$ ) the space $\mathcal{L}_{\omega}(0)$ provides a weighted version of $B M O$ larger than the one used by Muckenhoupt and Wheeden in [M-W1]. However, for the class of weights they obtained, that is $\omega^{\frac{n}{n-\alpha}} \in A_{1}$, both spaces coincide. When $1<p<n / \alpha$ (i.e. $\beta<0$ ), the space $\overline{\mathcal{L}}_{\omega}(\beta)$ contains the Lebesgue space $L^{q}, 1 / q=1 / p-\alpha / n$, as we showed in Proposition 1.8. Therefore, the 
analogue for $\overline{\mathcal{L}}_{\omega}(\beta)$ of Theorem 2.5 gives the boundedness of $I_{\alpha}$ from $L_{\omega}^{p}$ into a space larger than that in [M-W1] but, on the other hand, we obtained a larger class of weights.

(2.16) Remark. We would like to point out that Theorem 2.9 generalizes the classical unweighted result on the boundedness of $I_{\alpha}$ between $B M O$ and the Lipschitz space $\Lambda(\alpha)$. Moreover, we note that for weights in our class the spaces $\mathcal{L}_{\omega}(\beta), 0<\beta<1 / n$, coincide with the pointwise versions given in (1.5), because, as we shall see in the next section, they satisfy a doubling condition.

We postpone the proofs of the theorems until $\S 4$, since we need first to establish some properties for our weights.

\section{BASIC PROPERTIES OF THE WEIGHTS}

In order to prove some properties for the weights introduced in $\S 2$, we need two technical lemmas about real functions.

(3.1) Lemma. Let $\varphi$ be a non negative and non decreasing function defined on $(0, \infty)$. If there exist two positive constants $C$ and $r$ such that

$$
\int_{t}^{\infty} \frac{\varphi(s)}{s^{r+1}} d s \leq C \frac{\varphi(t)}{t^{r}}
$$

for every $t>0$, then the function $\varphi(t) / t^{r}$ is quasi-decreasing with constant equal to $C 2^{r+1}$, that is, for any $t_{1} \leq t_{2}, \varphi\left(t_{1}\right) / t_{1}^{r} \leq C 2^{r+1} \varphi\left(t_{2}\right) / t_{2}^{r}$.

Proof. Let $t_{1} \geq t_{2}$. From (3.2), since $\varphi$ is non-decreasing, we have

$$
\begin{aligned}
\frac{\varphi\left(t_{1}\right)}{t_{1}^{r}} & =2^{r+1} t_{1} \frac{\varphi\left(t_{1}\right)}{\left(2 t_{1}\right)^{r+1}} \leq 2^{r+1} \int_{t_{1}}^{2 t_{1}} \frac{\varphi(s)}{s^{r+1}} d s \\
& \leq 2^{r+1} C \frac{\varphi\left(t_{2}\right)}{t_{2}^{r}}
\end{aligned}
$$

as we wanted to prove. $\diamond$

(3.3) Lemma. Let $\varphi$ be as in Lemma 3.1. Then the following conditions are equivalent:

(3.4) The function $\varphi$ satisfies (3.2).

(3.5) There exists $a>1$ such that $\varphi(a t) \leq 2^{-1} a^{r} \varphi(t)$ for every $t>0$.

(3.6) There exist two positive constants $C$ and $\epsilon$ such that

$$
\varphi(\theta t) \leq C \theta^{r-\epsilon} \varphi(t)
$$

for all $t>0$ and all $\theta \geq 1$.

Proof. In order to prove that (3.4) implies (3.5), let $a$ be a constant greater than one such that $\log a=2^{r+2} C^{2}$. Now, assume that there exists $t_{0}>0$ such that 
$\varphi\left(a t_{0}\right)>2^{-1} a^{r} \varphi\left(t_{0}\right)$. Then, from (3.4) and Lemma 3.1, we get

$$
\begin{aligned}
C \frac{\varphi\left(t_{0}\right)}{t_{0}^{r}} & \geq \int_{t_{0}}^{a t_{0}} \frac{\varphi(s)}{s^{r+1}} d s \geq \frac{1}{C 2^{r+1}} \frac{\varphi\left(a t_{0}\right)}{\left(a t_{0}\right)^{r}} \log a \\
& >\frac{1}{C 2^{r+2}} \frac{\varphi\left(t_{0}\right)}{t_{0}^{r}} \log a=C \frac{\varphi\left(t_{0}\right)}{t_{0}^{r}},
\end{aligned}
$$

which is a contradiction. Let us show (3.5) implies (3.6). Let $\theta \geq 1$. Choosing $k \in \mathbf{N}_{0}$ such that $a^{k} \leq \theta<a^{k+1}$, and iterating the inequality in (3.5), we have

$$
\begin{aligned}
\varphi(\theta t) \leq \varphi\left(a^{k+1} t\right) & \leq \frac{a^{r}}{2} \varphi\left(a^{k} t\right) \leq\left(\frac{a^{r}}{2}\right)^{k+1} \varphi(t) \\
& \leq \frac{a^{r} \theta^{r}}{2^{k+1}} \varphi(t) \leq a^{r} \theta^{r} 2^{-\log _{2} \theta / \log _{2} a} \varphi(t) \\
& =a^{r} \theta^{r-\epsilon} \varphi(t),
\end{aligned}
$$

where $\epsilon=1 / \log _{2} a>0$, which finishes the proof of (3.6). Next, assume that (3.6) holds. Therefore, for $t>0$, we get

$$
\int_{t}^{\infty} \frac{\varphi(s)}{s^{r+1}} d s=\frac{1}{t^{r}} \int_{1}^{\infty} \frac{\varphi(\theta t)}{\theta^{r+1}} d \theta \leq C \frac{\varphi(t)}{t^{r}} \int_{1}^{\infty} \theta^{-(1+\epsilon)} d \theta \leq C \frac{\varphi(t)}{t^{r}},
$$

which proves $(3.4) . \diamond$

In the following we will search for properties valid for the classes $H(\alpha, p)$ with $0<\alpha<n$ and $1<p \leq \infty$.

(3.7) Lemma. Let $\omega$ be a weight belonging to $H(\alpha, p)$. Then $\omega^{p^{\prime}}$ satisfies a doubling condition.

Proof. Let $B$ be a ball in $\mathbb{R}^{n}$; since $\omega$ belongs to $H(\alpha, p)$, we have

$$
\begin{aligned}
& |B|^{(1 / p-\alpha / n+1 / n) p^{\prime}} \int_{\mathbb{R}^{n}} \frac{\omega^{p^{\prime}}(y)}{\left(\left|x_{B}-y\right|+|B|^{1 / n}\right)^{(n-\alpha+1) p^{\prime}}} d y \\
& \leq C\left(\frac{\omega(B)}{|B|}\right)^{p^{\prime}}+|B|^{(1 / p-\alpha / n+1 / n) p^{\prime}} \int_{B} \frac{\omega^{p^{\prime}}(y)}{\left(\left|x_{B}-y\right|+|B|^{1 / n}\right)^{(n-\alpha+1) p^{\prime}}} d y \\
& \quad \leq C\left(\frac{\omega(B)}{|B|}\right)^{p^{\prime}}+|B|^{(1 / p-1) p^{\prime}} \int_{B} \omega^{p^{\prime}}(y) d y \leq C \frac{\omega^{p^{\prime}}(B)}{|B|} .
\end{aligned}
$$

Therefore,

$$
\begin{aligned}
\frac{\omega^{p^{\prime}}(B)}{|B|} & \geq C|B|^{(1 / p-\alpha / n+1 / n) p \prime} \int_{2 B} \frac{\omega^{p \prime}(y)}{\left(\left|x_{B}-y\right|+|B|^{1 / n}\right)^{(n-\alpha+1) p^{\prime}}} d y \\
& \geq C|B|^{(1 / p-1) p^{\prime}} \omega^{p^{\prime}}(2 B) \\
& =C|B|^{-1} \omega^{p \prime}(2 B) .
\end{aligned}
$$

This completes the proof. $\diamond$ 
(3.8) Lemma. Let $\omega$ be a weight in $H(\alpha, p), 1<p<\infty$. Then $\omega$ belongs to $R H\left(p^{\prime}\right)$.

Proof. Let $B$ be a ball in $\mathbb{R}^{n}$. Since $\omega$ satisfies $H(\alpha, p)$, applying Lemma 3.7, we get

$$
\begin{aligned}
\frac{\omega(B)}{|B|} & \geq C|B|^{1 / p-\alpha / n+1 / n}\left(\int_{2 B-B} \frac{\omega^{p \prime}(y)}{\left|x_{B}-y\right|^{(n-\alpha+1) p \prime}} d y\right)^{1 / p \prime} \\
& \geq C|B|^{1 / p-1}\left(\omega^{p^{\prime}}(2 B-B)\right)^{1 / p^{\prime}} \\
& \geq C\left(\frac{\omega^{p^{\prime}}(B)}{|B|}\right)^{1 / p \prime}
\end{aligned}
$$

as we wished. $\diamond$

(3.9) Lemma. For a weight $\omega$ the following conditions are equivalent:

(3.10) $\omega$ belongs to $H(\alpha, p)$.

(3.11) $\omega$ belongs to $R H\left(p^{\prime}\right)$ and there exist two positive constants $C$ and $\epsilon$ such that

$$
\omega^{p^{\prime}}\left(B\left(x_{B}, \theta t\right)\right) \leq C \theta^{(n-\alpha+1) p^{\prime}-\epsilon} \omega^{p^{\prime}}\left(B\left(x_{B}, t\right)\right)
$$

for every ball $B=B\left(x_{B}, t\right)$ and for all $\theta \geq 1$.

There exist two positive constants $C$ and $\epsilon$ such that

$$
\left(\frac{\omega^{p^{\prime}}\left(B\left(x_{B}, \theta t\right)\right)}{\left|B\left(x_{B}, \theta t\right)\right|}\right)^{1 / p^{\prime}} \leq C \theta^{n / p-\alpha+1-\epsilon / p^{\prime}} \frac{\omega\left(B\left(x_{B}, t\right)\right)}{\left|B\left(x_{B}, t\right)\right|}
$$

for every ball $B\left(x_{B}, t\right)$ in $\mathbb{R}^{n}$ and for all $\theta \geq 1$.

Proof. Let us show (3.10) implies (3.11). By Lemma $3.8 \omega$ satisfies the desired reverse-Hölder inequality. On the other hand, for $B=B\left(x_{B}, t\right)$, using the Hölder inequality, (3.10) and Lemma 3.7, we have

$$
\begin{aligned}
\frac{\omega^{p^{\prime}}(B)}{|B|} & \geq C\left(\frac{\omega(B)}{|B|}\right)^{p^{\prime}} \\
& \geq C|B|^{(1 / p-\alpha / n+1 / n) p^{\prime}} \int_{\mathbb{R}^{n}-B} \frac{\omega^{p^{\prime}}(x)}{\left|x_{B}-x\right|^{(n-\alpha+1) p^{\prime}}} d x \\
& \geq C|B|^{(1 / p-\alpha / n+1 / n) p^{\prime}} \sum_{k=0}^{\infty} \frac{\omega^{p^{\prime}}\left(B\left(x_{B}, 2^{k+1} t\right)\right)}{\left(2^{k} t\right)^{(n-\alpha+1) p^{\prime}}} \\
& \geq C|B|^{(1 / p-\alpha / n+1 / n) p^{\prime}} \int_{t}^{\infty} \frac{\omega^{p^{\prime}}\left(B\left(x_{B}, s\right)\right)}{s^{(n-\alpha+1) p^{\prime}}} \frac{d s}{s} .
\end{aligned}
$$


Therefore, we get

$$
\int_{t}^{\infty} \frac{\omega^{p^{\prime}}\left(B\left(x_{B}, s\right)\right)}{s^{(n-\alpha+1) p^{\prime}}} \frac{d s}{s} \leq C \frac{\omega^{p^{\prime}}\left(B\left(x_{B}, t\right)\right)}{t^{(n-\alpha+1) p^{\prime}}}
$$

which implies $(3.2)$ for $\varphi(t)=\omega^{p^{\prime}}\left(B\left(x_{B}, t\right)\right)$ and $r=(n-\alpha+1) p^{\prime}$. Then, an application of Lemma 3.3 completes the proof of (3.11). Conversely, if (3.11) holds, by setting $\theta=2$ we get that $\omega^{p^{\prime}}$ satisfies a doubling condition. Therefore for a ball $B=B\left(x_{B}, t\right)$, we have

$$
\begin{aligned}
& \left(\int_{C B} \frac{\omega^{p^{\prime}}(x)}{\left|x_{B}-x\right|^{(n-\alpha+1) p^{\prime}}} d x\right)^{1 / p^{\prime}} \\
& \quad=\left(\sum_{k=0}^{\infty} \int_{2^{k} t<\left|x_{B}-x\right|<2^{k+1} t} \frac{\omega^{p^{\prime}}(x)}{\left|x_{B}-x\right|^{(n-\alpha+1) p^{\prime}}} d x\right)^{1 / p^{\prime}} \\
& \leq C\left(\sum_{k=0}^{\infty} \frac{\omega^{p^{\prime}}\left(B\left(x_{B}, 2^{k} t\right)\right)}{\left(2^{k} t\right)^{(n-\alpha+1) p^{\prime}}}\right)^{1 / p^{\prime}} \\
& \leq C\left(\omega^{p^{\prime}}(B)\right)^{1 / p^{\prime}}|B|^{\alpha / n-1 / n-1}\left(\sum_{k=0}^{\infty} 2^{-k \epsilon}\right)^{1 / p^{\prime}} \\
& \leq C \omega(B)|B|^{-1}|B|^{-1 / p+\alpha / n-1 / n} .
\end{aligned}
$$

This proves (3.10). Now let us assume (3.11). Then

$$
\begin{aligned}
\frac{\omega^{p^{\prime}}\left(B\left(x_{B}, \theta t\right)\right)}{\left|B\left(x_{B}, \theta t\right)\right|} & \leq C \theta^{(n-\alpha+1) p^{\prime}-n-\epsilon} \frac{\omega^{p^{\prime}}\left(B\left(x_{B}, t\right)\right)}{\left|B\left(x_{B}, t\right)\right|} \\
& \leq C \theta^{n\left(p^{\prime}-1\right)+(1-\alpha) p^{\prime}-\epsilon}\left(\frac{\omega\left(B\left(x_{B}, t\right)\right)}{\left|B\left(x_{B}, t\right)\right|}\right)^{p^{\prime}},
\end{aligned}
$$

which implies (3.12). Finally, taking $\theta=1$ in (3.12), we have that $\omega$ satisfies a reverse-Hölder inequality. On the other hand, from (3.12) and the Hölder inequality the estimate in (3.11) holds. This complete the proof of the lemma. $\diamond$

The following lemmas prove that for a fixed $\alpha$, the set of $p$ for which a given weight belongs to $H(\alpha, p)$ is an open interval.

(3.13) Lemma. Let $\omega$ be in $H(\alpha, p), 1<p<\infty$. Then there exists $\delta_{0} \in(0,1)$ such that $\omega$ belongs to $H\left(\alpha,\left(p^{\prime} \delta\right)^{\prime}\right)$ for any $\delta_{0} \leq \delta \leq 1$.

Proof. For any $\delta \in(0,1)$, from the Hölder inequality and Lemma 3.9, we have

$$
\left(\frac{\omega^{p^{\prime} \delta}\left(B\left(x_{B}, \theta t\right)\right)}{\left|B\left(x_{B}, \theta t\right)\right|}\right)^{1 / p^{\prime} \delta} \leq C \theta^{n /\left(p^{\prime} \delta\right)^{\prime}-\alpha+1-\tilde{\epsilon} /\left(p^{\prime} \delta\right)} \frac{\omega\left(B\left(x_{B}, t\right)\right)}{\left|B\left(x_{B}, t\right)\right|}
$$

for every ball $B\left(x_{B}, t\right)$ in $\mathbb{R}^{n}$ and for all $\theta \geq 1$, where

$$
\widetilde{\epsilon}=p^{\prime} \delta\left(\epsilon / p^{\prime}-n\left(1 / p+1 / p^{\prime} \delta-1\right)\right) .
$$


Now, choosing $\delta$ sufficiently close to one, we get $\widetilde{\epsilon}>0$. Thus, by Lemma 3.9 again, we obtain that $\omega$ is in $H\left(\alpha,\left(p^{\prime} \delta\right)^{\prime}\right) . \diamond$

(3.14) Lemma. Let $\omega$ be in $H(\alpha, p), 1<p<\infty$. Then there exist $\tau_{0}>1$ such that $\omega$ belongs to $H\left(\alpha,\left(p^{\prime} \tau\right)^{\prime}\right)$ for any $1 \leq \tau \leq \tau_{0}$.

Proof. From Lemma 3.8 and by Lemma 2 (p. 268) in [G], there exist $\tau_{0}>1$ and a positive constant $C$ such that for any $1 \leq \tau \leq \tau_{0}$

$$
\left(\frac{1}{|B|} \int_{B} \omega^{p^{\prime} \tau}\right)^{1 /\left(p^{\prime} \tau\right)} \leq C\left(\frac{1}{|B|} \int_{B} \omega^{p^{\prime}}\right)^{1 / p^{\prime}} \leq C \frac{\omega(B)}{|B|}
$$

for every ball $B$ in $\mathbb{R}^{n}$. Thus, for a given ball $B=B\left(x_{B}, t\right)$, by Lemma 3.7 and the $H(\alpha, p)$ condition, we have

$$
\begin{aligned}
\int_{\mathbb{R}^{n}-B} & \frac{\omega^{p^{\prime} \tau}(x)}{\left|x_{B}-x\right|^{(n-\alpha+1) p^{\prime} \tau}} d x=\sum_{k=0}^{\infty} \int_{2^{k} t \leq\left|x_{B}-x\right| \leq 2^{k+1} t} \frac{\omega^{p^{\prime} \tau}(x)}{\left|x_{B}-x\right|^{(n-\alpha+1) p^{\prime} \tau}} d x \\
& \leq \sum_{k=0}^{\infty}\left(2^{k} t\right)^{-(n-\alpha+1) p^{\prime} \tau} \int_{\left|x_{B}-x\right| \leq 2^{k+1} t} \omega^{p^{\prime} \tau}(x) d x \\
& \left.\leq C \sum_{k=0}^{\infty}\left(2^{k+1} t\right)^{n(1-\tau)}\left(2^{k} t\right)^{-(n-\alpha+1) p^{\prime}} \int_{\left|x_{B}-x\right| \leq 2^{k+1} t} \omega^{p^{\prime}}(x) d x\right)^{\tau} \\
& \leq C \sum_{k=0}^{\infty}\left(2^{k+1} t\right)^{n(1-\tau)}\left(\int_{2^{k} t \leq\left|x_{B}-x\right| \leq 2^{k+1} t} \frac{\omega^{p^{\prime}}(x)}{\left|x_{B}-x\right|^{(n-\alpha+1) p^{\prime}}} d x\right)^{\tau} \\
& \left.\leq C|B|^{1-\tau} \int_{\mathbb{R}^{n}-B} \frac{\omega^{p^{\prime}}(x)}{\left|x_{B}-x\right|^{(n-\alpha+1) p^{\prime}}} d x\right)^{\tau} \\
\leq & C\left(\frac{\omega(B)}{|B|}\right)^{p^{\prime} \tau}|B|^{p^{\prime} \tau\left(-1 /\left(p^{\prime} \tau\right)^{\prime}+\alpha / n-1 / n\right)} .
\end{aligned}
$$

This completes the proof of the lemma. $\diamond$

(3.15) Lemma. Let $1<p_{1}<p_{2} \leq \infty$. Assume that $\omega \in H\left(\alpha, p_{i}\right)$ for $i=1,2$. Then $\omega \in H(\alpha, p)$ for every $p$ such that $p_{1}<p<p_{2}$.

Proof. Let $\theta \in(0,1)$ and $1 / p=\theta / p_{1}+(1-\theta) / p_{2}$. Then $1 / p^{\prime}=\theta / p_{1}^{\prime}+(1-\theta) / p_{2}^{\prime}$, where $p^{\prime}=p /(p-1)$ and $p_{i}^{\prime}=p_{i} /\left(p_{i}-1\right)$ for $i=1,2$. Since $\omega \in H\left(\alpha, p_{i}\right)$, by the 
Hölder inequality, we have

$$
\begin{aligned}
& \left(\int_{\mathbb{R}^{n}-B} \frac{\omega^{p^{\prime}}(y)}{\left|x_{B}-y\right|^{(n-\alpha+1) p^{\prime}}}\right)^{1 / p^{\prime}} \\
& \quad=\left(\int_{\mathbb{R}^{n}-B} \frac{\omega^{\theta p \prime}(y)}{\left|x_{B}-y\right|^{(n-\alpha+1) \theta p^{\prime}}} \frac{\omega^{(1-\theta) p^{\prime}}(y)}{\left|x_{B}-y\right|^{(n-\alpha+1)(1-\theta) p^{\prime}}} d y\right)^{1 / p^{\prime}} \\
& \quad \leq\left(\int_{\mathbb{R}^{n}-B} \frac{\omega^{p_{1}}(y) d y}{\left|x_{B}-y\right|^{(n-\alpha+1) p_{1}^{\prime}}}\right)^{\theta / p_{1}^{\prime}}\left(\int_{\mathbb{R}^{n}-B} \frac{\omega^{p^{\prime}}(y) d y}{\left|x_{B}-y\right|^{(n-\alpha+1) p_{2}^{\prime}}}\right)^{(1-\theta) p_{2}^{\prime}} \\
& \quad \leq\left(|B|^{-1 / n+\alpha / n-1 / p_{1}-1} \omega(B)\right)^{\theta}\left(|B|^{-1 / n+\alpha / n-1 / p_{2}-1} \omega(B)\right)^{1-\theta} \\
& \quad=|B|^{-1 / n+\alpha / n-1 / p-1} \omega(B),
\end{aligned}
$$

which proves that $\omega \in H(\alpha, p) . \diamond$

(3.16) Examples of weights in $H(\alpha, p)$. It is easy to check that weights $\omega$ such that $\omega^{p^{\prime}} \in A_{1}$, are in $H(\alpha, p)$. However, the weights $w(x)=|x|^{\beta}$ with $\beta \in(0, n / p-$ $\alpha+1$ ) do not belong to $A_{1}$ but, using (3.9), it is easy to check that they are in $H(\alpha, p)$.

Let $0<\alpha<1$ and $p^{\prime}>n /(1-\alpha)$. The weights $\omega$ such that $\omega^{p^{\prime}} \in A_{p^{\prime}+1}$ are in $H(\alpha, p)$. In fact, given a ball $B$ and $\theta \geq 1, \omega^{p^{\prime}} \in A_{p^{\prime}+1}$ and Hölder inequality imply

$$
\begin{aligned}
\left(\frac{\omega^{p^{\prime}}(\theta B)}{|\theta B|}\right)^{1 / p^{\prime}} & \leq C \frac{|\theta B|}{\omega^{-1}(\theta B)} \leq C \frac{\theta^{n}|B|}{\omega^{-1}(B)} \\
& \leq C \theta^{n} \frac{\omega(B)}{|B|}=C \theta^{n / p-\alpha+1-\epsilon / p \prime} \frac{\omega(B)}{|B|}
\end{aligned}
$$

where $\epsilon=p^{\prime}\left(1-\alpha-n / p^{\prime}\right)>0$. From Lemma 3.9, we get $\omega \in H(\alpha, p)$. $\diamond$

\section{Proofs of the Main Results}

We start by proving some technical lemmas needed in the proof of our main results.

(4.1) Lemma. Let $w$ be a weight satisfying a reverse Hölder condition with exponent $p^{\prime}, 1<p<\infty$. Let $f$ belong to the weak weighted $L^{p}$-space $\tilde{L}_{w}^{p}$. Then, there exists a constant $C$, independent of $f$, such that

$$
\int_{B}|f(x)| d x \leq C \frac{w(B)}{|B|^{1 / p}}[f]_{p, w}
$$

for every ball $B$ in $\mathbb{R}^{n}$. 
Proof. Given a ball $B$, since $\omega \in R H\left(p^{\prime}\right)$, by Lemma 2 (p. 268) in [G], there exist $\delta>0$ and a constant $C$ such that

$$
\left(\frac{1}{|B|} \int_{B} \omega^{p^{\prime}+\delta}(x) d x\right)^{1 /\left(p^{\prime}+\delta\right)} \leq C \frac{\omega(B)}{|B|} .
$$

Therefore, by the Hölder inequality, we get

$$
\begin{aligned}
\int_{B}|f(x)| d x & \leq\left(\int_{B} \omega^{p^{\prime}+\delta}(x) d x\right)^{1 /\left(p^{\prime}+\delta\right)}\left(\int_{B}\left(\frac{|f(x)|}{\omega(x)}\right)^{\left(p^{\prime}+\delta\right)^{\prime}} d x\right)^{1 /\left(p^{\prime}+\delta\right)^{\prime}} \\
& \leq C \omega(B)\left(\frac{1}{|B|} \int_{B}\left(\frac{|f(x)|}{\omega(x)}\right)^{q} d x\right)^{1 / q}
\end{aligned}
$$

where $q=\left(p^{\prime}+\delta\right)^{\prime}$. Let $a$ be a constant to be determined later. We now estimate

$$
\begin{aligned}
\int_{B}\left(\frac{|f(x)|}{\omega(x)}\right)^{q} d x & \leq q \int_{0}^{a} t^{q-1}\left|\left\{\frac{|f|}{\omega}>t\right\} \cap B\right| d t+q \int_{a}^{\infty} t^{q-1}\left|\left\{\frac{|f|}{\omega}>t\right\}\right| d t \\
& \leq|B| a^{q}+q[f]_{p, \omega}^{p} \int_{a}^{\infty} t^{q-p-1} d t \\
& =|B| a^{q}+q[f]_{p, \omega}^{p} \frac{a^{q-p}}{p-q}
\end{aligned}
$$

because $q<p$. Thus, choosing $a=[f]_{p, \omega}|B|^{-1 / p}$, we obtain

$$
\int_{B}\left(\frac{|f(x)|}{\omega(x)}\right)^{q} d x \leq C|B|^{1-q / p}[f]_{p, \omega}^{q}
$$

Thus, from (4.2) and (4.3), the conclusion of the lemma follows. $\diamond$

(4.4) Lemma. Let $\omega$ be in $H(\alpha, p), 1<p<\infty$. Let $f$ belong to $\tilde{L}_{\omega}^{p}$. Then, there exists a constant $C$, independent of $f$, such that

$$
\int_{\mathbb{R}^{n}-B} \frac{f(y)}{\left|x_{B}-y\right|^{n-\alpha+1}} d y \leq C \frac{\omega(B)}{|B|^{1+1 / n+1 / p-\alpha / n}}[f]_{p, \omega}
$$

for every ball $B=B\left(x_{B}, t\right)$ in $\mathbb{R}^{n}$.

Proof. Let $a$ be a constant to be determined later. Let us denote

$$
f^{a}=f \chi_{\left\{\frac{|f|}{\omega}>a\right\}} \quad \text { and } \quad f_{a}=f-f^{a} .
$$


Then, for a ball $B$ with center $x_{B}$, we have

$$
\begin{aligned}
& \int_{\mathbb{R}^{n}-B} \frac{f(y)}{\left|x_{B}-y\right|^{n-\alpha+1}} d y \\
& \quad=\int_{\mathbb{R}^{n}-B} \frac{f^{a}(y)}{\left|x_{B}-y\right|^{n-\alpha+1}} d y+\int_{\mathbb{R}^{n}-B} \frac{f_{a}(y)}{\left|x_{B}-y\right|^{n-\alpha+1}} d y \\
& \quad=I_{1}+I_{2} .
\end{aligned}
$$

Let us first estimate $I_{1}$. Since $\omega$ is in $H(\alpha, p)$, by Lemma 3.14, there exists $\tau>1$ such that $\omega \in H\left(\alpha, q_{1}\right)$ with $q_{1}=\left(p^{\prime} \tau\right)^{\prime}<p$. Then, applying the Hölder inequality, we get

$$
\begin{aligned}
\left|I_{1}\right| & \leq\left(\int_{\mathbb{R}^{n}-B} \frac{\omega^{q_{1}^{\prime}}(y)}{\left|x_{B}-y\right|^{(n-\alpha+1) q_{1}^{\prime}}} d y\right)^{1 / q_{1}^{\prime}}\left(\int_{\mathbb{R}^{n}}\left|\frac{f^{a}(y)}{\omega(y)}\right|^{q_{1}} d y\right)^{1 / q_{1}} \\
& \leq C \frac{\omega(B)}{|B|^{1+\frac{1}{n}+\frac{1}{q_{1}}-\frac{\alpha}{n}}}\left(q_{1} \int_{0}^{\infty} s^{q_{1}-1}\left|\left\{\left|\frac{f^{a}}{\omega}\right|>s\right\}\right| d s\right)^{1 / q_{1}} \\
& \leq C \frac{\omega(B)}{|B|^{1+\frac{1}{n}+\frac{1}{q_{1}}-\frac{\alpha}{n}}}\left(a^{q_{1}}\left|\left\{\left|\frac{f^{a}}{\omega}\right|>a\right\}\right|+q_{1}[f]_{p, \omega}^{p} \int_{a}^{\infty} s^{q_{1}-p-1} d s\right)^{1 / q_{1}} \\
& \left.\leq C \frac{\omega(B)}{|B|^{1+\frac{1}{n}+\frac{1}{q_{1}}-\frac{\alpha}{n}}}\left(a^{q_{1}-p}\right)[f]_{p, \omega}^{p}\right)^{1 / q_{1}} .
\end{aligned}
$$

On the other hand, by Lemma 3.13, there exists $0<\delta<1$ such that $\omega \in H\left(\alpha, q_{2}\right)$ with $q_{2}=\left(p^{\prime} \delta\right)^{\prime}>p$. Thus, by the Hölder inequality, it follows that

$$
\begin{aligned}
\left|I_{2}\right| & \leq\left(\int_{\mathbb{R}^{n}-B} \frac{\omega^{q_{2}^{\prime}}(y)}{\left|x_{B}-y\right|^{(n-\alpha+1) q_{2}^{\prime}}} d y\right)^{1 / q_{2}^{\prime}}\left(\int_{\mathbb{R}^{n}}\left|\frac{f_{a}(y)}{\omega(y)}\right|^{q_{2}} d y\right)^{1 / q_{2}} \\
& \leq C \frac{\omega(B)}{|B|^{1+1 / n+1 / q_{2}-\alpha / n}}\left(\int_{0}^{a} s^{q_{2}-1}\left|\left\{\frac{|f|}{\omega}>s\right\}\right| d s\right)^{1 / q_{2}} \\
& \leq C \frac{\omega(B)}{|B|^{1+1 / n+1 / q_{2}-\alpha / n}}\left(a^{q_{2}-p}[f]_{p, \omega}^{p}\right)^{1 / q_{2}} .
\end{aligned}
$$

Now, choosing $a=[f]_{p, \omega}|B|^{-\frac{1}{p}}$, from (4.5) and (4.6) the proof of the lemma is complete. $\diamond$

(4.7) Lemma. Let $\alpha \in \mathbb{R}^{+}$and $\delta \geq 0$ be such that $0<\alpha+\delta<1$. Let $\omega$ be a weight satisfying a doubling condition. If $f$ belongs to $\mathcal{L}_{\omega}(\delta / n)$, then there exists a 
constant $C$ such that

$$
\int_{\mathbb{R}^{n}-B} \frac{\left|f(y)-m_{B} f\right|}{\left|x_{B}-y\right|^{n+1-\alpha}} d y \leq C\|f\|_{\mathcal{L}_{\omega}(\delta / n)} \int_{\mathbb{R}^{n}-B} \frac{\omega(y)}{\left|x_{B}-y\right|^{n+1-\alpha-\delta}} d y
$$

for every ball $B$ in $\mathbb{R}^{n}$.

Proof. Given a ball $B=B\left(x_{B}, R\right)$, letting $B_{j}=2^{j} B$, since $\omega$ satisfies a doubling condition, we have

$$
\begin{aligned}
& \int_{\mathbb{R}^{n}-B} \frac{\left|f(y)-m_{B} f\right|}{\left|x_{B}-y\right|^{n+1-\alpha}} d y \\
& \leq C|B|^{\frac{\alpha-1}{n}} \sum_{j=0}^{\infty} 2^{j(\alpha-1)} \frac{1}{\left|B_{j+1}\right|} \int_{B_{j+1}-B_{j}}\left|f(y)-m_{B} f\right| d y \\
& \leq C|B|^{\frac{\alpha-1}{n}} \sum_{j=0}^{\infty} 2^{j(\alpha-1)} \sum_{k=0}^{j+1} \frac{1}{\left|B_{k}\right|} \int_{B_{k}}\left|f(y)-m_{B_{k}} f\right| d y \\
& \leq C\|f\|_{\mathcal{L}_{\omega}(\delta / n)}|B|^{\frac{\alpha-1}{n}} \sum_{j=0}^{\infty} 2^{j(\alpha-1)} \sum_{k=0}^{j+1} \frac{\omega\left(B_{k}\right)}{\left|B_{k}\right|^{1-\frac{\delta}{n}}} \\
& \leq\left. C|| f\right|_{\mathcal{L}_{\omega}(\delta / n)}|B|^{\frac{\alpha-1}{n}} \sum_{k=0}^{\infty} \frac{\omega\left(B_{k}\right)}{\left|B_{k}\right|^{1-\frac{\delta}{n}}} 2^{k(\alpha-1)} \\
& \leq C\|f\|_{\mathcal{L}_{\omega}(\delta / n)} \int_{\mathbb{R}^{n}-B} \frac{\omega(y)}{\left|x_{B}-y\right|^{n+1-\alpha-\delta}} d y,
\end{aligned}
$$

as we wanted to prove. $\diamond$

The results obtained in $\S 3$ and the above lemmas give us the needed tools to proceed with the proofs of theorems we stated in $\S 2$.

Proof of Theorem 2.5. Clearly (2.7) implies (2.6). Assuming (2.8), we shall prove (2.7). In order to extend $I_{\alpha}$ to an operator $\tilde{I}_{\alpha}$, we first note that if $f$ belongs to $\tilde{L}_{\omega}^{p}$ and has compact support, then from Lemma 4.1 it follows easily that $I_{\alpha} f(x)$ is finite for almost every $x$ in $\mathbb{R}^{n}$. Now, letting $\chi_{B(0,1)}(y)$ denote the characteristic function of the unit ball, the operator

$$
\tilde{I}_{\alpha} f(x)=\int_{\mathbb{R}^{n}}\left[\frac{1}{|x-y|^{n-\alpha}}-\frac{1-\chi_{B(0,1)}(y)}{|y|^{n-\alpha}}\right] f(y) d y
$$

is well defined for every $f$ in $\tilde{L}_{\omega}^{p}$. In fact, for a ball $B=B\left(x_{B}, r\right)$ with $\tilde{B}=2 B$, we set

$$
a_{B}=\int_{\mathbb{R}^{n}}\left(\frac{1-\chi_{\tilde{B}}(y)}{\left|x_{B}-y\right|^{n-\alpha}}-\frac{1-\chi_{B(0,1)}(y)}{|y|^{n-\alpha}}\right) f(y) d y .
$$

The expression in parentheses is bounded and behaves like $\left|x_{B}-y\right|^{-n+\alpha-1}$ for large $y$. Then, since $\omega$ is in $H(\alpha, p)$, applying Lemmas 4.1 and 4.4 , we get that $a_{B}$ is 
finite. Thus, if we are able to show that

$$
\begin{aligned}
I(x) & =\int_{\tilde{B}} \frac{f(y)}{|x-y|^{n-\alpha}} d y+\int_{\mathbb{R}^{n}-\tilde{B}}\left(\frac{1}{|x-y|^{n-\alpha}}-\frac{1}{\left|x_{B}-y\right|^{n-\alpha}}\right) f(y) d y \\
& =I_{1}(x)+I_{2}(x) .
\end{aligned}
$$

satisfies the inequality

$$
\int_{B}|I(x)| d x \leq C|B|^{\alpha / n-1 / p} \omega(B)[f]_{p, \omega},
$$

then, since by breaking the integral defining $a_{B}$ into the sum of the integrals over $\widetilde{B}$ and its complement it is easy to check that

$$
\widetilde{I}_{\alpha} f(x)=a_{B}+I(x),
$$

we get that $\widetilde{I}_{\alpha} f$ is finite for almost every $x$ in $B$ and

$$
\int_{B}\left|\widetilde{I}_{\alpha} f(x)-a_{B}\right| d x \leq C|B|^{\alpha / n-1 / p} \omega(B)[f]_{p, \omega},
$$

which implies (2.7). Let us first estimate $I_{1}(x)$. From Tonelli's Theorem and Lemma 4.1, we obtain

$$
\int_{B}\left|I_{1}(x)\right| d x \leq C|B|^{\alpha / n-1 / p} \omega(B)[f]_{p, \omega} \quad .
$$

On the other hand, by Lemma 4.4, for each $x \in B$, we have

$$
\begin{aligned}
\left|I_{2}(x)\right| & \leq C|B|^{1 / n} \int_{\mathbb{R}^{n}-\tilde{B}} \frac{f(y)}{\left|x_{B}-y\right|^{n-\alpha+1}} d y \\
& \leq C|B|^{-1-1 / p+\alpha / n} \omega(B)[f]_{p, \omega} .
\end{aligned}
$$

When we combine these estimates, the inequality for $I(x)$ follows immediately.

Finally, let us show (2.6) implies (2.8). First, for a ball $B=B\left(x_{B}, R\right)$ in $\mathbb{R}^{n}$ and for $\tilde{x}_{B}=x_{B}-\frac{R}{3 \sqrt{n}}(1,1, \ldots, 1)$, we consider the following three regions:

$$
\begin{aligned}
& A=\left\{x_{B}+h \text { with } h \in \mathbb{R}^{n} /|h| \geq R \text { and } h_{i} \geq 0, i=1, \ldots, n\right\} ; \\
& B_{1}=B\left(x_{B}, \frac{R}{6 \sqrt{n}}\right) \cap\left\{x_{B}+h \text { with } h \in \mathbb{R}^{n} / h_{i} \leq 0, i=1, \ldots, n\right\} ; \\
& B_{2}=B \cap\left\{\tilde{x}_{B}+h \text { with } h \in \mathbb{R}^{n} / h_{i} \leq 0, i=1, \ldots, n\right\} .
\end{aligned}
$$

Clearly, we have the estimates

$$
\begin{aligned}
& \left|B_{1}\right|=2^{-n}\left|B\left(x_{B}, \frac{R}{6 \sqrt{n}}\right)\right|=C(n) R^{n}=C(n)|B|, \\
& \left|B_{2}\right| \geq 2^{-n}\left|B\left(\tilde{x}_{B}, \frac{2}{3} R\right)\right|=C(n) R^{n}=C(n)|B|, \\
& \operatorname{dist}\left(B_{1}, B_{2}\right) \geq R\left(\frac{1}{3}-\frac{1}{6 \sqrt{n}}\right)=C(n)|B|^{\frac{1}{n}} .
\end{aligned}
$$


Then, for $y \in A, x \in B_{1}$ and $z \in B_{2}$, applying the mean value theorem and denoting the angle between two vectors $u$ and $v$ by $(\widehat{u, v})$, we get

$$
\frac{1}{|x-y|^{n-\alpha}}-\frac{1}{|z-y|^{n-\alpha}}=(-n+\alpha) \frac{\cos (w-\widehat{y, x}-z)}{|w-y|^{n-\alpha+1}}|x-z|,
$$

where $w$ is a point in the segment conecting $x$ and $z$. Under these conditions, it follows that there exists $\theta=\theta(n) \in\left(\frac{\pi}{2}, \pi\right)$ such that $(w-\widehat{y, x}-z) \geq \theta$, and, consequently, $-\cos (w-\widehat{y, x}-z) \geq C=C(n)$. Therefore, for $y \in A, x \in B_{1}$ and $z \in B_{2}$, since $|w-y| \leq 2\left|x_{B}-y\right|$, we have

$$
\frac{1}{|x-y|^{n-\alpha}}-\frac{1}{|z-y|^{n-\alpha}} \geq C \frac{|x-z|}{|w-y|^{n-\alpha+1}} \geq \frac{C|B|^{1 / n}}{\left|x_{B}-y\right|^{n-\alpha+1}} .
$$

Now, given a ball $B$ and a non negative function $f_{m}$ with support in $A_{m}=$ $A \cap B(0, m), m \in \mathbb{N}$, from (1.1) with $\beta=\alpha / n-1 / p$ and (4.8), we get

$$
\begin{aligned}
& \left\|I_{\alpha} f_{m}\right\|_{\mathcal{L}_{\omega}(\beta)} \\
& \quad \geq \frac{|B|^{1 / p-\alpha / n-1}}{\omega(B)} \int_{B_{2}} \int_{B_{1}}\left|\int_{A_{m}} f_{m}(y)\left(\frac{1}{|x-y|^{n-\alpha}}-\frac{1}{|z-y|^{n-\alpha}}\right) d y\right| d x d z \\
& \quad \geq C \frac{|B|^{1+1 / p-\alpha / n+1 / n}}{\omega(B)} \int_{A_{m}} \frac{f_{m}(y)}{\left|x_{B}-y\right|^{n-\alpha+1}} d y .
\end{aligned}
$$

Thus, taking

$$
f_{m}(y)=\frac{\omega^{p^{\prime}}(y)}{\left|x_{B}-y\right|^{\frac{n-\alpha+1}{p-1}}} \chi_{A_{m}}(y)
$$

in (4.9), using (2.6) and letting $m \rightarrow \infty$, we have that

$$
|B|^{(1 / p-\alpha / n+1 / n) p^{\prime}} \int_{A} \frac{\omega^{p^{\prime}}(y)}{\left|x_{B}-y\right|^{(n-\alpha+1) p^{\prime}}} d y \leq C\left(\frac{\omega(B)}{|B|}\right)^{p^{\prime}} .
$$

Let us observe that $A$ is the complement of $B$ relative to the first quadrant of the Cartesian system with center at $x_{B}$. Proceeding as above, with the complement of $B$ with respect to the other quadrants, we get similar estimates for each of these regions. By adding all these inequalities we conclude that $\omega$ is in $H(\alpha, p)$, completing the proof of the theorem. $\diamond$

Proof of Theorem 2.9. Assume (2.11). First we shall see that $I_{\alpha}$ can be extended to an operator $\tilde{I}_{\alpha}$ defined on $\mathcal{L}_{\omega}(0)$. Since

$$
\begin{aligned}
\int_{B(0,1)}\left(\int_{B(x, R)} \frac{\omega(y)}{|x-y|^{n-\alpha}} d y\right) d x & \leq \int_{B(0, R+1)} \omega(y)\left(\int_{B(0,1)} \frac{d x}{|x-y|^{n-\alpha}}\right) d y \\
& \leq C(n, \alpha) \int_{B(0, R+1)} \omega(y) d y<\infty
\end{aligned}
$$


for every $R \in \mathbb{R}_{+}$, we can choose $x_{0} \in B(0,1)$ such that

$$
\int_{B\left(x_{0}, R\right)} \frac{\omega(y)}{\left|x_{0}-y\right|^{n-\alpha}} d y<\infty \quad \text { for every } R \in \mathbb{R}_{+} .
$$

Thus, for $x_{0}$ as above, we set

$$
\tilde{I}_{\alpha} f(x)=\int_{\mathbb{R}^{n}}\left(\frac{1}{\left|x_{0}-y\right|^{n-\alpha}}-\frac{1}{|x-y|^{n-\alpha}}\right) f(y) d y .
$$

Since the expression in parentheses has null integral over $\mathbb{R}^{n}$ as a function of $y$, for $B=B\left(x_{0}, R\right)$, we get

$$
\begin{aligned}
\tilde{I}_{\alpha} f(x) & =\int_{\mathbb{R}^{n}}\left(\frac{1}{\left|x_{0}-y\right|^{n-\alpha}}-\frac{1}{|x-y|^{n-\alpha}}\right)\left(f(y)-m_{B} f\right) d y \\
& =I_{1}(x)+I_{2}(x),
\end{aligned}
$$

where $I_{1}$ is the integral over the ball $B$ and $I_{2}$ is the integral on the complement of $B$. Let us first estimate $I_{1}$ for $x \in B\left(x_{0}, R\right)$. We set $\tilde{B}=B(x, 2 R)$. Since, by Lemma 3.7, $\omega$ satisfies a doubling condition, we have

$$
\begin{aligned}
\left|I_{1}(x)\right| \leq & \int_{B} \frac{\left|f(y)-m_{B} f\right|}{\left|x_{0}-y\right|^{n-\alpha}} d y+\int_{\tilde{B}} \frac{\left|f(y)-m_{B} f\right|}{|x-y|^{n-\alpha}} d y \\
\leq & \int_{B} \frac{\left|f(y)-m_{B} f\right|}{\left|x_{0}-y\right|^{n-\alpha}} d y+\int_{\tilde{B}} \frac{\left|f(y)-m_{\tilde{B}} f\right|}{|x-y|^{n-\alpha}} d y \\
& +\left.C|| f\right|_{\mathcal{L}_{\omega}(0)}|B|^{\alpha / n-1} \omega(B) .
\end{aligned}
$$

Both integrals can be estimated in exactly the same way, so we do only the first one. Thus, denoting $B_{k}=2^{-k} B, k \in \mathbb{N}$, we get 


$$
\begin{aligned}
\int_{B} \frac{\left|f(y)-m_{B} f\right|}{\left|x_{0}-y\right|^{n-\alpha}} d y & \leq C|B|^{\frac{\alpha}{n}} \sum_{k=0}^{\infty} 2^{-k \alpha}\left|B_{k}\right|^{-1} \int_{B_{k}-B_{k-1}}\left|f(y)-m_{B} f\right| d y \\
& \leq C|B|^{\frac{\alpha}{n}} \sum_{k=0}^{\infty} 2^{-k \alpha} \sum_{j=0}^{k}\left|B_{j}\right|^{-1} \int_{B j}\left|f(y)-m_{B_{j}} f\right| d y \\
& \leq C|| f \|_{\mathcal{L}_{\omega}(0)}|B|^{\frac{\alpha}{n}} \sum_{k=0}^{\infty} 2^{-k \alpha} \sum_{j=0}^{k}\left|B_{j}\right|^{-1} \omega\left(B_{j}\right) \\
& \leq C|| f \|_{\mathcal{L}_{\omega}(0)}|B|^{\frac{\alpha}{n}-1} \sum_{j=0}^{\infty} 2^{j n} \omega\left(2^{-j} B\right) \sum_{k=j}^{\infty} 2^{-k \alpha} \\
& \leq C|| f \|_{\mathcal{L}_{\omega}(0)}|B|^{\frac{\alpha}{n}-1} \sum_{j=0}^{\infty} 2^{-j n\left(\frac{\alpha}{n}-1\right)} \omega\left(2^{-j} B\right) \\
& \leq C\|f\|_{\mathcal{L}_{\omega}(0)} \int_{B} \frac{\omega(y)}{\left|x_{0}-y\right|^{n-\alpha}} d y .
\end{aligned}
$$

Therefore

$$
I_{1} \leq C|| f \|_{\mathcal{L}_{\omega}(0)}\left(\int_{B} \frac{\omega(y)}{\left|x_{0}-y\right|^{n-\alpha}} d y+\int_{\tilde{B}} \frac{\omega(y)}{|x-y|^{n-\alpha}} d y\right) .
$$

Next, let us estimate $I_{2}$. Applying the mean value theorem and Lemma (4.7) with $\delta=0$, since $\omega \in H(\alpha / n, \infty)$, we have

$$
\begin{aligned}
I_{2} & \leq C|B|^{\frac{1}{n}} \int_{\mathbb{R}^{n}-B} \frac{\left|f(y)-m_{B} f\right|}{\left|x_{0}-y\right|^{n+1-\alpha}} d y \\
& \leq\left. C|| f\right|_{\mathcal{L}_{\omega}(0)}|B|^{\frac{1}{n}} \int_{\mathbb{R}^{n}-B} \frac{\omega(y)}{\left|x_{0}-y\right|^{n+1-\alpha}} d y \\
& \leq\left. C|| f\right|_{\mathcal{L}_{\omega}(0)}|B|^{\frac{\alpha}{n}-1} \omega(B) \\
& \leq C|| f \|_{\mathcal{L}_{\omega}(0)} \int_{B} \frac{\omega(y)}{\left|x_{0}-y\right|^{n-\alpha}} d y .
\end{aligned}
$$

By integrating on $B\left(x_{0}, R\right)$, from (4.10), (4.12) and (4.13), we obtain that the function $\tilde{I}_{\alpha} f(x)$ is finite for almost every $x$ in $B\left(x_{0}, R\right)$. Then, since $\mathbb{R}^{n}=$ $\bigcup_{R \in \mathbb{Q}^{+}} B\left(x_{0}, R\right)$, we can conclude that $\tilde{I}_{\alpha} f(x)$, given in (4.11), is finite for almost every $x \in \mathbb{R}^{n}$. 
Now, in order to prove the boundedness of $\tilde{I}_{\alpha}$ we observe that by Proposition 1.3 and (3.8) it is enough to get for $I_{\alpha} f$ a pointwise estimate as in (1.6). Given $x_{1}$ and $x_{2}$ in $\mathbb{R}^{n}$ with $x_{1} \neq x_{2}$ and $B=B\left(x_{1}, 2\left|x_{1}-x_{2}\right|\right)$, since the kernel of $\tilde{I}_{\alpha} f(x)$ has a null integral, we have

$$
\begin{aligned}
\left|\tilde{I}_{\alpha} f\left(x_{1}\right)-\tilde{I}_{\alpha} f\left(x_{2}\right)\right| & \leq \int_{\mathbb{R}^{n}}\left|\frac{1}{\left|x_{1}-y\right|^{n-\alpha}}-\frac{1}{\left|x_{2}-y\right|^{n-\alpha}}\right|\left|f(y)-m_{B} f\right| d y \\
& =I_{1}+I_{2},
\end{aligned}
$$

where $I_{1}$ is the integral on $B$ and $I_{2}$ is the integral on the complement of $B$. Thus, proceeding in a similar way as in (4.11) and (4.13), we get that

$$
\begin{aligned}
& \left|\tilde{I}_{\alpha} f\left(x_{1}\right)-\tilde{I}_{\alpha} f\left(x_{2}\right)\right| \\
& \quad \leq C\|f\|_{\mathcal{L}_{\omega}(0)}\left(\int_{B\left(x_{1}, 2\left|x_{1}-x_{2}\right|\right)} \frac{\omega(y)}{\left|x_{1}-y\right|^{n-\alpha}} d y+\int_{B\left(x_{2}, 2\left|x_{1}-x_{2}\right|\right)} \frac{\omega(y)}{\left|x_{2}-y\right|^{n-\alpha}} d y\right),
\end{aligned}
$$

as we wanted. Next, to prove (2.10) implies (2.11), let us proceed as in the proof of Theorem 2.5.

Choose the same sets $A, B_{1}$ and $B_{2}$ for a ball $B$ in $\mathbb{R}^{n}$. Then, for $m \in \mathbb{N}$, taking $f_{m}(y)=\omega(y) \chi_{A_{m}}(y)$, with $A_{m}=A \cap B(0, m)$, we get

$$
\begin{aligned}
\left\|\tilde{I}_{\alpha} f_{m}\right\|_{\mathcal{L}_{\omega}(\alpha / n)} & =\left\|I_{\alpha} f_{m}\right\|_{\mathcal{L}_{\omega}(\alpha / n)} \\
& \geq C \frac{|B|^{1+1 / n-\alpha / n}}{\omega(B)} \int_{A_{m}} \frac{\omega(y)}{\left|x_{B}-y\right|^{n-\alpha+1}} d y .
\end{aligned}
$$

Since $\left\|f_{m}\right\|_{\mathcal{L}_{\omega}(0)} \leq 2$, from $(2.10)$ we have

$$
|B|^{1 / n-\alpha / n} \int_{A_{m}} \frac{\omega(y)}{\left|x_{B}-y\right|^{n-\alpha+1}} d y \leq C \frac{\omega(B)}{|B|} .
$$

Thus, letting $m \rightarrow \infty$ and arguing as in Theorem 2.5, we obtain $w \in H(\alpha, \infty)$. This complete the proof of the theorem. $\diamond$

Proof of Corollary 2.12. Let us show (2.13) implies (2.14). First, since $\omega \in H(\delta, \infty)$, from Theorem 2.9, we have $I_{\delta}\left(L_{c, \omega}^{\infty}\right) \subset \mathcal{L}_{\omega}(\delta / n)$. On the other hand, by $(0.2)$, we get

$$
\left|I_{\alpha}\left(I_{\delta}(g)\right)(x)\right|=\left|I_{\alpha+\delta}(g)(x)\right|<\infty \quad \text { a.e. }
$$

for every $g$ in $L_{c, \omega}^{\infty}$. Then, the operator $\tilde{I}_{\alpha}$ agrees with $I_{\alpha}$ on $I_{\delta}\left(L_{c, \omega}^{\infty}\right)$. Finally, from this fact, (2.13) and Theorem 2.9, for $f \in L_{c, \omega}^{\infty}$, we have that

$$
\begin{aligned}
\left\|\tilde{I}_{\alpha}\left(I_{\delta} f\right)\right\|_{\mathcal{L}_{\omega}((\delta+\alpha) / n)} & =\left\|I_{\alpha+\delta}(f)\right\|_{\mathcal{L}_{\omega}((\delta+\alpha) / n)} \\
& \leq C\left\|\tilde{I}_{\delta} f\right\|_{\mathcal{L}_{\omega}(\alpha / n)} \\
& \leq C\|f\|_{\mathcal{L}_{\omega}(0)} .
\end{aligned}
$$

Thus, proceeding as in the proof of (2.11), from (2.10) we get (2.14). Now, assume (2.14). Clearly this implies $\omega \in H(\delta, \infty)$. Arguing as above, we have $I_{\delta}\left(L_{c, \omega}^{\infty}\right) \subset$ 
$\mathcal{L}_{\omega}(\delta / n)$ and $I_{\alpha}$ is defined on this set. In order to obtain an extension to all $\mathcal{L}_{\omega}(\delta / n)$, we choose a point $x_{0}$ as in (4.10) but replacing $\alpha$ by $\alpha+\delta$. Then, proceeding as in the proof of (2.10) in Theorem 2.9 but using (2.14) instead of (2.11), we define the extended operator $\tilde{I}_{\alpha}$ as in (4.11). The boundedness of this operator follows from an argument quite similar to that used in the proof of (2.10), but applying this time (2.14) instead of (2.8). $\diamond$

\section{REFERENCES}

[F-M] Fefferman, C. and Muckenhoupt, B. "Two Nonequivalent Conditions for Weight Functions" Proc. Amer. Math. Soc. 45, pp. 99-104. (1974) MR 53:13399

[G] Gehring, F. W. "The $L^{p}$-Integrability of the Partial Derivatives of a Quasiconformal Mapping" Acta Math. 130, pp 265-277. (1973) MR 53:5861

[GC] García Cuerva, J. "Weighted $H^{p}$ Spaces" Dissertationes Math. (Rozprawy Nat.) No. 162. (1979) MR 82a:42018

[G-V] Gatto, A. and Vagi, S. "Fractional Integrals on Spaces of Homogeneous Type". Analysis and Partial Differential Equations, Lecture Notes in Pure and Appl. Math., vol. 122, Marcel Dekker, New York, 1990, pp. 171-216. MR 91e:42032

[H-M-W] Hunt, R.; Muckenhoupt, B. and Wheeden, R. "Weighted norm inequalities for the conjugate function and Hilbert transform" Trans. Amer. Math. Soc. 176, pp. 227251. (1973) MR 47:701

[H-S-V] Harboure, E.; Salinas, O. and Viviani, B. "Acotación de la Integral Fraccionaria en Espacios de Orlicz y de Oscilación Media $\Phi$-Acotada" Actas del II Congreso Dr. A. Monteiro, Univ. Nac. del Sur, Bahía Blanca, pp.41-50. (1993) MR 95b:47036

[M] Muckenhoupt, B. "Weighted Norm Inequalities for Classical Operators" Harmonic Analysis in Euclidean Spaces, Proc. Sympos. Pure Math., vol. 35, part 1, Amer. Math. Soc., Providence, RI, pp. 69-84. (1979) MR 80i:42015

[M-W1] Muckenhoupt, B. and Wheeden, R. "Weighted Norm Inequalities for Fractional Integrals". Trans. Amer. Math. Soc. 192, pp. 261-274. (1974) MR 49:5275

[M-W2] Muckenhoupt, B. and Wheeden, R. "Weighted Bounded Mean Oscillation and the Hilbert Transform". Studia Math. 54 pp. 221-237. (1976) MR 53:3583

[P] Peetre, J. "On the theory of $\mathcal{L}_{p, \lambda}$ Spaces" J. Funct. Anal. 4, pp.71-87.(1969) MR 39:3300

[S-Z] Stein, E. M. and Zygmund, A. "Boundedness of Translation Invariant Operators on Hölder Spaces and L $L^{p}$ Spaces" Ann. of Math. 85, pp. 337-349. (1967) MR 35:5964

[Z] Zygmund, A. "Trigonometric Series" Vols. I, II, 2nd. Rev., Cambridge Univ. Press. (1958) MR 21:6498

Programa Especial de Matemática Aplicada and Facultad de Ingeniería Química, Universidad Nacional del Litoral, Güemes 3450, 3000 Santa Fe, Rep. Argentina 